\title{
DESIGN APPROACH TO IMPLEMENT IMPLICIT TRAFFIC IN A SIMULATION ENVIRONMENT
}

\author{
Charles A. Brooks \\ Aaron Chesir \\ Erica Lindy \\ Jon A. McConnell \\ The MITRE Corporation \\ Eatontown, NJ, U.S.A.
}

\author{
Monica Farah-Stapleton \\ U.S. Army Communications-Electronics Research \\ Development and Engineering Center (CERDEC) \\ Fort Monmouth, NJ, U.S.A.
}

\begin{abstract}
As the United States' Army attempts to spiral future force technologies into the current force, realistic representations of network-centric warfare become a priority for modeling and simulation (M\&S). Specifically, future communications systems will provide different performance and capabilities than current systems, and these new capabilities need to be represented by the Army's M\&S. In order to enable realistic analysis, the new communications systems models will need to be loaded by realistic representations of traffic flows to ensure the accuracy of the command, control, communications, computers, intelligence, surveillance, reconnaissance (C4ISR) analysis results. This paper will present a design approach and a series of algorithms to implement implicit traffic in the Future Combat Systems (FCS) Simulation Environment (FSE). The paper will focus on a phased approach for the generation of implicit traffic that will realistically load the modeled communications networks as the level of explicit traffic within the simulation environment increases from a small percentage to a large percentage of overall traffic.
\end{abstract}

\section{INTRODUCTION}

Current US Army battle simulations do not generate enough explicit traffic (the set of traffic generated by, or on behalf of, the simulated entities in the environment), preventing realistic loading of the modeled command, control, communications, computers, intelligence, surveillance, and reconnaissance (C4ISR) networks. This lack of realistic network loading prevents meaningful analysis of the evolving C4ISR network architectures. Methodologies and algorithms need to be developed to generate the appropriate implicit, or background, traffic (the set of traffic not explicitly generated by, or on behalf of, the simulated entities in an environment, but that would be operationally generated in a real-world environment), based on the force structure, the scenario being played, and the unit operations in simulation, as well as the current level of explicit traffic being modeled within the simulation architecture. One critical goal that this methodology must meet is to ensure a seamless transition over time from a small percentage of explicitly generated traffic to a high percentage. (Lindy and McConnell 2005).

\subsection{Definitions and Terminologies}

This paper is focused on Warfighter-generated traffic, which is traffic induced by simulated entities within a simulation environment. The methodology and approach presented in this paper does not address other types of traffic that exist in the simulation environment, such as simulation-platform-specific middleware and network management traffic.

Warfighter-Generated Traffic can be implicit or explicit, as defined as follows:

- Explicit Traffic: The set of traffic generated by, or on behalf of, the simulated entities in an environment AND sent over the simulation network via a specific message format with well defined fields.

- Implicit Traffic: The set of traffic not explicitly generated by, or on behalf of, the simulated entities in an environment, but that would be operationally generated in a real-world environment.

Warfighter-Generated implicit traffic can be modeled in the following ways:

- Discrete vs. Continuous:

- Discrete: Message-based.

- Continuous: Flow-based.

- Atomized vs. Aggregated:

- Atomized: Information Exchange Requirements (IERs) from the Department of Defense Architecture Framework (DoDAF) Operational View-3 (OV3) are the atomic unit. 
- Aggregated: Messages from the IERs are aggregated as a time-varying flow for each Sender/Receiver/Priority (S/R/P) tuple.

- Scheduled vs. Frequency-Based vs. Event-Driven:

- Scheduled: Specific start times for each IER.

- Frequency-Based: Start times for each IER are based on a stochastic process model.

- Event-Driven: Messages are triggered by events in the simulation environment.

\section{IMPLICIT TRAFFIC OPTIONS}

The accuracy of a force-on-force battle simulation and the corresponding analysis depends upon the inclusion of the effects of real-time communications among the nodes in the simulation. The two most relevant characteristics of each message exchange among the nodes are the completion/incompletion of the message and the delay of the message. Therefore, in a force-on-force battle simulation, it is necessary to determine, for each message, if the message from a source arrived at each intended destination and the delay of each completed message.

It is not always feasible in a simulation to explicitly instantiate each and every message among the nodes in the simulation. Therefore the sum of all messages explicitly instantiated in the simulation and the assumed implicit or background traffic that is not explicitly instantiated in the simulation needs to be equal to the total communications traffic among the nodes in the simulation. The purpose of an implicit traffic algorithm is to generate a representation of the implicit traffic for presentation to the communications and networking models used in the simulation.

For the methodology and algorithms described in this paper, the completion/delay characteristics of the implicit messages do not need to be determined, but the presence of these messages serves to load the communications links of the nodes in the simulation and therefore has a directly relevant effect upon the completion/delay characteristics of the explicit messages.

There are five options for representing/generating the implicit traffic, and each can be derived from an algorithm, as follows (Brooks and. Chesir 2005).

\subsection{Option I: Discrete / Atomized / Scheduled}

In this option, the implicit traffic is represented by actual messages with a sender, receiver, and length. Each implicit message is presented to the communications and networking models at a pre-determined point in time.

\subsection{Option II: Discrete / Atomized / Frequency-Based}

Like Option I, the implicit traffic in Option II is represented by actual messages with a specific sender, receiver, and length. However, for Option II each implicit message is presented to the communications and networking models at a point in time, based upon the message repetition rate specified in the IER database. The time of transmission is dithered by a stochastic process model (e.g., Poisson distribution).

Option II offers the possibility of running much of the algorithm (the Poisson process) in the communications and networking models, to reduce the traffic between the implicit traffic modeler and the communications and networking models.

\subsection{Option III: Discrete / Aggregated / Frequency- Based}

In this option, the implicit traffic is also represented by messages with a specific sender, receiver, and, length. As opposed to the prior two options, in option III, the presented messages simply convey the total traffic load from sender to receiver implied by the IER database. The total load is divided into messages with lengths that aggregate the load of all the messages listed in the IERs between the sender and receiver within a pre-determined, quantized interval of time. As with Option II, each presented message is presented to the communications and networking models at a user-settable message repetition rate and process model (e.g., Poisson distribution). By setting the message repetition rate, the implicit traffic modeler adjusts the message sizes so that the presented traffic load, in bytes, is the same as that implied by the IER database.

As with Option II, this option offers the possibility of running much of the algorithm in the communications and networking models, to reduce the traffic between the implicit traffic modeler and the communications and networking models.

\subsection{Option IV: Continuous / Aggregated / Frequency- Based}

In this option, the implicit traffic is modeled not by individual messages, but rather by a specification of the bit rate of implicit traffic for every sender and receiver. This bit rate may change at each quantized interval of time.

The resulting implicit flow specification table is sorted by time so that the implicit traffic modeler can step through the table of flows in step with the passage of simulation time. The information in this table would be used by the communications and networking models to modulate the capacity available for explicit traffic among the senderreceiver pairs.

\subsection{Option V: Discrete / Atomized / Event-Driven}

This option is a modification of Options 1 or II above. The implicit traffic modeler must have real-time access to indi- 
cations of simulation events that imply the subsequent generation of otherwise implicit messages. When the implicit traffic modeler detects such events, it presents each consequent implicit message to the communications and networking models. All other implicit messages are handled in the normal Option I or II approach.

This option requires that the algorithm has, as an input, a representation of the cause-effect model between certain simulation events and the consequent implicit messages.

\section{WARFIGHTER IMPLICIT TRAFFIC GENERATION DESIGN}

The implicit traffic design approach is to be implemented in 2 steps, as follows:

1. A Communications Traffic Load Generator (CTLG) which will use a discrete/atomized approach (option II) that is frequency-driven.

2. A Warfighter-Generated Implicit Traffic Approach (WarGen ITA), which is the combination of the CTLG (for implicit traffic that is not in response to simulation events) and an algorithm that listens for simulation events for which there is a cause/effect relationship between the event and consequent implicit traffic (option V). When such events are detected, the WarGen ITA responds with the generation of the appropriate implicit traffic.

\subsection{Communications Traffic Load Generator (CTLG)}

The CTLG will run an Option II algorithm, which will be IER-based and force structure-specific. Option II was chosen because it has the following characteristics:

- Moderate Degree of Fidelity.

- Smallest Degree of algorithm development.

- Moderate degree of IER generation effort.

- Low amount of traffic between the implicit traffic modeler and the communications and networking models.

- Moderate amount of real-time computational load.

\subsubsection{Option II Algorithm}

Though the IER database itself does not contain timestamps (these are synthesized later when associating the IERs to a scenario), the Option II algorithm generates a schedule for the firing of the implicit messages using a statistical frequency distribution, based upon the message repetition rate (which is a parameter of each entry in the IER database). A Poisson process is used for this purpose.
In any IER data set, there are certain messages that are marked as "forced to fire," and all other messages are therefore presumed "unforced to fire." The identification of "forced to fire" means that the message is scheduled based strictly upon its associated message repetition rate, and not subject to the vagaries of stochastic (e.g., Poisson) processes. Messages that are forced to fire are guaranteed to be scheduled within the time period of the phase of the battle. In the Option II Algorithm, there is a parameter $\alpha$ (ALPHA) whose purpose is strictly to artificially increase or decrease the implicit traffic load of the simulation. This does not imply a change in the tempo of the battle being simulated.

\subsection{Phase 1b - WarGen ITA Overview}

The WarGen ITA will be a new component and will run the Option V algorithm (discrete/atomized/event-driven) for a specified set of operational domains, and the Option II algorithm for all other implicit traffic. The Option V algorithm will be a modified version of the Option II algorithm, augmented with real-time simulation triggers from an interactive, high resolution, entity-level simulation (currently the One Semi-Automated Forces (OneSAF) Test Bed Baseline (OTB)), and in the future, the OneSAF Objective System (OOS).

\subsubsection{Event-Driven Terms}

In order to derive different design alternatives, the following WarGen ITA terms were defined:

- Generic Traffic: Traffic not linked to a force structure or scenario, but represents all possible requirements based on operational behaviors.

- Connected Traffic: Generic traffic linked to a particular force structure, but not to a particular scenario.

- $\quad$ Scheduled Traffic: Traffic linked to the events in a particular scenario and a particular force structure, and, therefore, the IERs represented are only those relevant to the sender's and receiver's events in that particular scenario.

- Sequenced Traffic: Message traffic occurs in a pre-determined order.

- Non-Sequenced Traffic: Message traffic is sent in a stochastic order, but still in the context of operational behaviors via a domain (i.e., Networked Fires).

\subsubsection{Triggers and Alternatives}

There are three criteria associated with event-driven implicit traffic: 
- Connected Traffic vs. Scheduled Traffic.

- Sequenced Traffic vs. Non-Sequenced Traffic.

- Number of Triggers.

Different combinations of the above criteria will result in event-driven design alternatives. The chosen approach was to use Connected/Sequenced Mission Threads. Mission Threads are represented in a DoDAF operational view (OV-6) that sequences IERs in the context of a mission. Within the mission thread sequencing there are many IERs, and as a result, the Option V Algorithm will pre-determine the time intervals between sequenced traffic stochastically, bounded by the total amount of time it would take to complete the operations, not the communications traffic estimate. In addition, each OTB trigger is associated with a simulated entity, and the simulated entity's echelon will be used as part of the intensity factor within the algorithm.

\subsubsection{Option V Algorithm}

The purpose of the Option V algorithm is to provide implicit warfighter-generated traffic to the communications and networking models in response to the detection of events in the simulation. This algorithm has not been designed; however, it will be the Option II algorithm augmented with event triggers. Before the simulation run, a cause/effect model of the messages that result from each of the recognized events during the simulation is needed to initialize the Option V Algorithm.

During simulation runtime, the following are needed to initialize the Option V Algorithm:

- Indications of relevant battle events.

- Constant updates on the status of the members of the force structure.

- Constant updates on the mapping between members of the force structure and the nodes identified in the mission threads.

- Constant updates to the simulation time.

- Appropriately-timed heartbeat indications from the communications and networking models.

\subsubsection{Data Requirement}

The same data elements used to initialize the Option II algorithm are needed to initialize the Option V Algorithm, with the addition of Event OR (Event AND Sequence).

\subsubsection{Transition to Explicit Traffic}

One critical goal that this methodology must meet is to ensure a seamless transition over time from a small percentage of explicitly generated traffic to a high percentage. The development of the CTLG and associated Option II algorithm is an important step in the phased approach because the event-driven approach will only look at a few opera- tional domains at a time. Therefore, the CTLG will be used for all other background traffic not modeled as eventdriven. As the WarGen ITA models different domains in detail (i.e., Networked Fires, Logistics, etc.) and the associated triggers are available within the simulation environment, future study teams will be able to recommend new triggers needed in order to model the domain focus with operational realism. This recommendation will be handed-off to the simulation community, who will transition from the original set of implicit traffic available in the simulation to increased explicit traffic in future simulation version releases.

\section{IMPACT TO THE FCS SIMULATION ENVIRONMENT (FSE)}

Within FSE 1.0, the computer generated forces are the only components that currently produce explicit messages. As a result, a small subset of traffic will be event-driven at any given time. After researching the FCS program's "Spin Out 1" configuration, as shown in Figure 1, it was determined that Networked Fires, Intelligent Munitions System (IMS)/Unattended Ground Sensors (UGS), and Logistics should be the first operational domains modeled as eventdriven background traffic in the WarGen ITA component. The advantage of focusing on these domains first is due to their key roles in Spin Out 1. Also, there exists data on all three domains that can be used to initialize the Option V algorithm and these areas, specifically Networked Fires, have been the focus of many simulation studies to date which could provide the data necessary for the development of new event triggers.

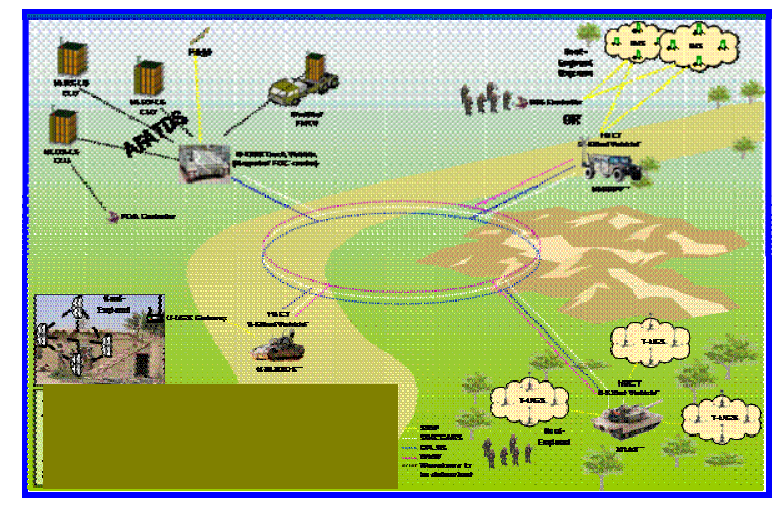

Figure 1: The Spin Out 1 Configuration

In addition to the computer generated forces, test articles, which are hardware prototypes under development by the FCS Lead Systems Integrator (LSI), can be introduced in the FSE architecture and produce explicit traffic, as shown in Figure 2. The Tactical Message Communications Effects Server (CES) Interface (TMCI) is both hardware and software that resides between the test articles and the 
CES in the FSE 1.0 Architecture. In order for a test article to communicate with another component, its messages are intercepted by the TMCI and held while the CES calculates the communications performance. The TMCI then forwards the message to the original intended target or, in the case of message failure (determined by CES), discards the message (Prochnow et al. 2005).

Explicit traffic enters the simulation environment as a trigger, as shown in Figure 2. In the FSE 1.0 architecture, the OTB explicit message first is interpreted in the Federation Object Model (FOM). If the OTB message is not defined in the FOM, the OTB message is disregarded. If the message is defined in the FOM, then the message is sent to the Communications Grid (C3Grid). The C3Grid either responds with a C3Grid defined situation report (SITREP) or Size, Activity, Location, Unit, Time, Equipment (SALUTE) report, in the case of OTB entity perceived state (EPS) interactions, to one or many recipients, or the C3Grid passes the message through unaltered. Thus, the FOM acts as a condition point in the explicit message flow in FSE 1.0 and will need to be enhanced with additional OTB generated messages to allow for explicit traffic already present in OTB/OOS. The $\mathrm{C} 3 \mathrm{Grid}$ routes all messages to the CES for communications performance data.

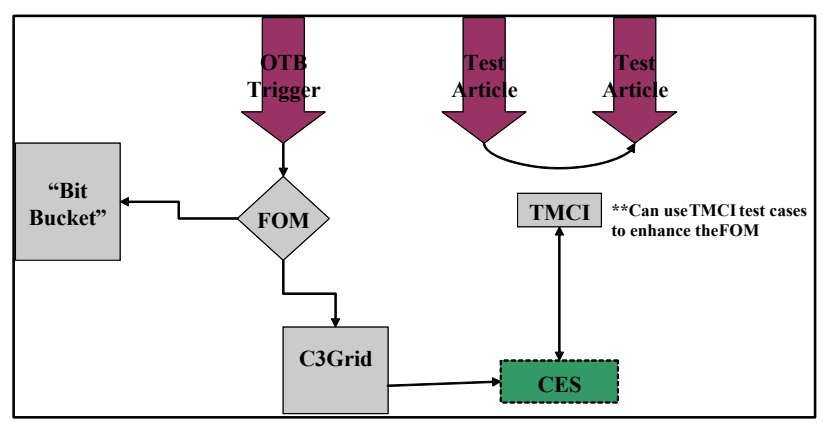

Figure 2. Message Flow of Explicit Traffic in FSE 1.0

\section{CONCLUSION}

The phased design approach of implementing the frequency-driven CTLG in the near-term, followed by the addition of the event-driven WarGen ITA component, provides a feasible, low-risk solution for the generation of implicit traffic in a simulation. The use of this implementation, associated data, and algorithms will realistically load the C4ISR networks within the US Army's simulation environments and allow for a seamless transition over time to an increase of explicitly generated traffic as a result of an increase of operational behavior models.

\section{REFERENCES}

Lindy, E., and J. McConnell. 2005. Strawman Design Approach to Implement Warfighter-Generated Implicit
Traffic in the FCS Simulation Environment (FSE) 1.0, Final Report. Army Communications-Electronics Research Development and Engineering Center (CERDEC) HQs, December 2005.

Prochnow, D., M. Fogus, J. Vintelescu, and B. Borum. 2005. Initialization of Distributed Simulations: A Better Way? In Proceedings of the Fall 2005 Simulation Interoperability Workshop.

Brooks, C. A., and A. Chesir. 2005. CASTFOREM Background Traffic White Paper- Version 1.0, Final Report published on behalf of the Army CommunicationsElectronics Research Development and Engineering Center (CERDEC) HQs.

\section{AUTHOR BIOGRAPHIES}

CHARLES A. BROOKS has worked for The MITRE Corporation, for the past 7 years, where he is a Technical Manager in the Modeling \& Simulation and Data Analysis Group within the Integrated Communications, Networks and Sensors Department. During this tenure, he has served as technical lead for several modeling and simulation (M\&S) efforts, including the design and implementation of methodologies for incorporating communications effects within the Army's suite of combat simulation models for the purpose of modeling C4ISR for Current and Future Forces. He has also led the development of a hybrid modeling and simulation-based analysis resource for the Army Chief Information Officer (CIO/G6) in support of CSA directives for Army Transformation of the Current Force. Charles obtained his Ph.D. in Electrical Engineering and Computer Sciences and M.S. in Electrical Engineering and Computer Sciences from the University of California, Berkeley and a B.S. in Computer and Systems Engineering from Rensselaer Polytechnic Institute. His e-mail address is <cbrooksamitre. org $>$.

AARON CHESIR has over 20 years of experience in the design and implementation of communications systems. This includes DSP algorithm design and implementation, circuit design, FPGA design, and software development. Since joining MITRE, Aaron has been involved in ForceOn-Force modeling and simulation. Aaron received his Bachelor of Engineering from The Cooper Union in 1983, and his Master of Engineering in 1984. His e-mail address is <achesir@mitre.org $>$.

MONICA FARAH-STAPLETON is currently the Program Director for Modeling and Simulation (M\&S) at the Army Communications-Electronics Research Development and Engineering Center (CERDEC) HQs, at Ft. Monmouth. She is responsible for coordinating crossorganization integration of C4ISR M\&S experimentation, focusing on the representations of C4ISR technologies and concepts in models of varying fidelity, e.g., physics-based 
models through Force-on-Force simulations. Her previous 2-year assignment was as the Project Leader directing the CERDEC C4ISR System of Systems engineering analyses for the Future Force. Ms. Farah-Stapleton has been an invited speaker at numerous conferences and has presented M\&S/Architecture initiatives to senior Army and DoD leadership. She has a BSEE from Rutgers University, and an Executive Masters of Science in Engineering from the University of Pennsylvania. Her e-mail address is $<$ Monica.Farahstapleton@us . army.mil>.

ERICA LINDY is currently a Senior Modeling and Simulation Engineer for the MITRE Corporation and has supported the U. S. Army Communications-Electronics Research, Development and Engineering Center (CERDEC) HQ at Ft. Monmouth, New Jersey for the past 5 years. Her focus is in Command and Control, Communications, Computers and Intelligence, Surveillance, and Reconnaissance (C4ISR) analyses from a system of systems perspective. She received her Mathematics degree at Rutgers College, Rutgers University of New Jersey. Her e-mail address is <elindyamitre.org>.

JON A. McCONNELL is currently The MITRE Corporation project leader for support to the Air Force Space Command Director of Requirements in Colorado Springs. He spent his previous 18 years at MITRE supporting the U. S. Army at Fort Monmouth, New Jersey. His previous position was in support of the Communications-Electronics Research, Development and Engineering Center (CERDEC) Architecture and Systems Engineering Office (ASEO). His e-mail address is <jmcconne@mitre. org $>$. 